\title{
ISLA AKTUELL: DREI INSPIRIERENDE ÜBERSICHTSARBEITEN ZUR PHOTOBIOMODULATION
}

\section{Liebe Kolleginnen und Kollegen,}

die ISLA hat sich zum Ziel gesetzt, nicht nur Pionierforschung durchzuführen, sondern auch höchstrangige Veröffentlichungen zu bewerkstelligen. So wurden beispielsweise während der COVID-19-Lockdown-Phasen unter der Erstautorenschaft von Frau Professor Laura Ailioaie und unter der Beteiligung des ISLA-Präsidenten Gerhard Litscher drei Übersichtsartikel mit insgesamt 116 Druckseiten und einem Impact Faktor von 13,668 in einem angesehenen Journal der Molekularwissenschaften veröffentlicht. Bislang wurden auf die Open-Access-Publikationen bereits ca. 5000 Zugriffe gezählt, was für so spezielle Review-Arbeiten in sehr kurzer Zeit ebenfalls einen hohen Wert darstellt.

Vorteile und Perspektiven der angewandten sogenannten Photobiomik für die Gesundheit werden in der ersten Arbeit aufgezeigt. Gekoppelte, komplexe Photobiomodulation- (PBM-) und probiotische Interventionen könnten die Aktivität des Immunsystems verbessern und das Leben von Menschen mit Immunschwächen beispielsweise in der Coronavirus-Krise erfolgreich retten (Ailioaie LM, Litscher G. Probiotics, Photobiomodulation, and Disease Management: Controversies and Challenges. Int J Mol Sci. 2021; 22(9): 4942).

Wie hinlänglich bekannt, kann Curcumin als Naturprodukt kostengünstig und ohne Nebenwirkungen bestimmte Bereiche des menschlichen Immunsystems sehr gut stimulieren. Curcumin und die blaue Photobiomodulation könnenViren unterdrücken und bestimmte Krankheiten besser kontrollieren, indem sie Entzündungen reduzieren und das Fortschreiten etwa einer chronischen
Hepatitis stoppen. Die zweite Publikation zielt auf diese Fragestellung (Ailioaie LM, Litscher G. Curcumin and Photobiomodulation in Chronic Viral Hepatitis and Hepatocellular Carcinoma. Int J Mol Sci. 2020;21(19): 7150).

Juvenile idiopathische Arthritis und adulte rheumatoide Arthritis sind zwei Hauptgruppen mit chronischen Gelenkschmerzen und Entzündungen, extraartikulären Manifestationen und einem hohen Risiko für Komorbiditäten, die zu körperlichen Behinderungen führen und weltweit einen hohen sozioökonomischen Druck erzeugen können. Die PBM stellt auch hier eine gute Option dar und bietet kostengünstige Vorteile gegenüber der medikamentösen Therapie mit einem schnelleren, positiveren Ansprechen auf die Behandlung. Lesen Sie mehr in der dritten Übersichtsarbeit (Ailioaie LM, Litscher G. Molecular and Cellular Mechanisms of Arthritis in Children and Adults: New Perspectives on Applied Photobiomodulation. Int J Mol Sci. 2020; 21 (18): 6565).

Licht ist ein sehr leistungsfähiges Tool in der Medizin, da es imVergleich zu Medikamenten gleichzeitig auf viele Kaskaden der Aktivierung des Immunsystems abzielen kann. Die PBM und auch die Laserakupunktur können sehr heikle Aufgaben in unseren Zellen ausführen, um zelluläre Funktionsstörungen zu modulieren, Selbstorganisationsphänomene auszulösen und schließlich Krankheiten zu lindern bzw. unter Umständen zu heilen.

Allen Leser`innen dieser Ausgabe wünschen wir weiterhin Gesundheit und alles Gute.

G. Litscher

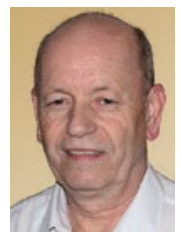

Dr. med. Dipl. Chem. Michael Weber

President for Medical and Clinical Applications der International Society for Medical Laser Applications (ISLAtranscontinental)

Sohnreystrasse 4

37697 Lauenförde

Internet: www.isla-laser.org

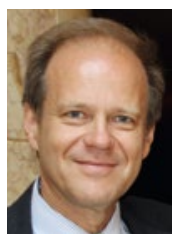

Univ.-Prof. Prof. h.c. DI Dr. techn.

Dr. scient. med. Gerhard Litscher

President for Science and Research der International Society for Medical Laser Applications (ISLAtranscontinental)

Medizinische Universität Graz, Auenbruggerplatz 39, EG19, A-8036 Graz, Tel: +43 316/385-83907 E-Mail:gerhard.litscher@medunigraz.at 